\title{
Bayesian predictions for $A=6$ nuclei using eigenvector continuation emulators
}

\author{
T. Djärv, ${ }^{*}$ A. Ekström, C. Forssén $\odot,{ }^{\dagger}$ and H. T. Johansson $\odot$ \\ Department of Physics, Chalmers University of Technology, SE-412 96 Göteborg, Sweden
}

(Received 1 September 2021; accepted 10 January 2022; published 26 January 2022)

\begin{abstract}
We make ab initio predictions for the $A=6$ nuclear level scheme based on two- and three-nucleon interactions up to next-to-next-to-leading order in chiral effective field theory $(\chi$ EFT). We utilize eigenvector continuation and Bayesian methods to quantify uncertainties stemming from the many-body method, the $\chi$ EFT truncation, and the low-energy constants of the nuclear interaction. The construction and validation of emulators is made possible via the development of JUPITERNCSM - a new $M$-scheme no-core shell model code that uses on-the-fly Hamiltonian matrix construction for efficient, single-node computations up to $N_{\max }=10$ for ${ }^{6} \mathrm{Li}$. We find a slight underbinding of ${ }^{6} \mathrm{He}$ and ${ }^{6} \mathrm{Li}$, although consistent with experimental data given our theoretical error bars. As a result of incorporating correlated $\chi$ EFT-truncation errors we find more precise predictions (smaller error bars) for separation energies: $S_{d}\left({ }^{6} \mathrm{Li}\right)=0.89 \pm 0.44 \mathrm{MeV}, S_{2 n}\left({ }^{6} \mathrm{He}\right)=0.20 \pm 0.60 \mathrm{MeV}$, and for the beta decay $Q$ value: $Q_{\beta^{-}}\left({ }^{6} \mathrm{He}\right)=3.71 \pm 0.65 \mathrm{MeV}$. We conclude that our error bars can potentially be reduced further by extending the model space used by JUPITERNCSM.
\end{abstract}

DOI: 10.1103/PhysRevC.105.014005

\section{INTRODUCTION}

The use of ab initio many-body solvers and effective field theory (EFT) descriptions of nuclear interactions promises to deliver rigorous uncertainty quantification in the theoretical modeling of low-energy nuclear observables. The key advantage is that this combination makes it possible to systematically quantify the magnitude of errors made when approximating the solution of the many-body problem and when modeling the nuclear interaction to a finite EFT order.

A Bayesian framework is propitious for carrying out such an uncertainty quantification program. Best practices for using Bayesian methods in the context of EFT descriptions have been presented in, e.g., Refs. [1-6]. Recently, such methods have also been applied in the context of many-nucleon systems from posterior sampling in the few-body sector [6,7], to estimates of EFT truncation errors in light nuclei [8] and infinite nuclear matter $[9,10]$. However, the full incorporation of pertinent sources of uncertainties in computationally expensive many-body predictions is challenging. In this paper we present both new computational technology and wellmotivated statistical models for relevant error terms to take first steps towards a comprehensive analysis of uncertainties in $a b$ initio nuclear structure theory.

The goals of this paper can be summarized as follows:

\footnotetext{
*djarv@ chalmers.se

†christian.forssen@chalmers.se
}

Published by the American Physical Society under the terms of the Creative Commons Attribution 4.0 International license. Further distribution of this work must maintain attribution to the author $(s)$ and the published article's title, journal citation, and DOI. Funded by Bibsam.
(1) Use Bayesian methods to demonstrate the propagation of parametric (statistical) uncertainties of the threenucleon force (3NF) low-energy constants (LECs) $c_{D}$ and $c_{E}$ to $A=6$-body systems using a newly developed no-core shell model (NCSM) many-body solver.

(2) Construct and sample statistical models for both (many-body) method and (EFT) model uncertainties to obtain the posterior predictive distribution (PPD) for a set of many-body observables.

(3) Introduce a new open-source, NCSM computer code-JUPITERNCSM [11] - that is is designed to solve relatively large many-body problems on single compute nodes such that it becomes possible to efficiently explore a large set of Hamiltonian parametrizations.

(4) Construct and demonstrate the accuracy of eigenvector continuation (EC) emulators $[12,13]$ for $A=6$ NCSM observables.

We start in Sec. II with a description of a recent Bayesian inference analysis [6] of nuclear interaction parameters conditioned on calibration data in the two- and few-nucleon sector. The many-body calculations are presented in Sec. III where we also introduce the JUPITERNCSM code and construct EC emulators. Our uncertainty quantification for nuclear observables is summarized in PPDs as discussed in Sec. IV. Here we also develop statistical models to link our theoretical models with reality in order to make final predictions. We conclude in Sec. V with a summary and outlook.

\section{INTERACTION MODEL WITH STATISTICAL CONSTRAINTS}

Wesolowski et al. [6] recently inferred posterior probability density functions (PDFs) for the interaction LECs in chiral effective field theory $(\chi$ EFT) at leading order $(\mathrm{LO})$, nextto-leading order (NLO), and next-to-next-to-leading order 
(N2LO) of the chiral expansion. In particular, they analyzed the constraints on the leading $3 \mathrm{NF}$ that appears at N2LO from several few-nucleon observables: the ${ }^{3} \mathrm{H}$ and ${ }^{4} \mathrm{He}$ binding energies, the ${ }^{4} \mathrm{He}$ charge radius and the Gamow-Teller matrix element extracted from tritium $\beta$ decay. Henceforth we label this set of model calibration observables as $\mathcal{D}$. The parameter estimation considered both experimental and computational uncertainties as well as the model discrepancy that originates in the truncation of the $\chi$ EFT Hamiltonian. The latter was included using a statistical error model $[4,14]$ that relies on two parameters: the EFT expansion parameter $Q$ and the scale $\bar{c}$ of observable coefficients. The latter governs the magnitude of relative corrections at each $\chi$ EFT order to a single observable according to

$$
y_{\mathrm{th}}=y_{\mathrm{ref}} \sum_{n} c_{n} Q^{n}
$$

where it is also assumed that all coefficients $c_{n}$ are independent and identically distributed (i.i.d.) random variables following a normal distribution with zero mean and variance $\bar{c}^{2}: c_{n} \sim \mathcal{N}\left(0, \bar{c}^{2}\right)$. We will revisit this error model in Sec. IV B where we incorporate correlated truncation errors for a vector of observable predictions.

The vector of LEC parameters is collectively denoted $\vec{a}$. It includes a subset of elements $\vec{a}_{\mathrm{NN}}$ that govern the strengths of the two-nucleon force (2NF) contact interactions and a subset $\vec{a}_{3 \mathrm{~N}}$ that parametrize the strengths of the shorter-range diagrams of the leading $3 \mathrm{NF}$ appearing at N2LO in Weinberg power counting. At this order $\vec{a}_{3 \mathrm{~N}}$ consists of $c_{D}$ and $c_{E}$, the uncertainties of which are a main focus of this paper. Starting at $\mathrm{N} 2 \mathrm{LO}$, the $\chi \mathrm{EFT}$ interaction also includes longer-range pion-nucleon $(\pi \mathrm{N})$ interactions parametrized by $\vec{a}_{\pi \mathrm{N}}$. In this work, we fix the corresponding LECs at mean values determined in a Roy-Steiner analysis of $\pi \mathrm{N}$ scattering data [15].

The analysis by Wesolowski et al. [6] was performed with a nonlocal momentum-space regulator function as in Eqs. (5) and (6) of Ref. [16] with a single cutoff $\Lambda=450 \mathrm{MeV}$ and $n=3$. The $\vec{a}_{\mathrm{NN}}$ LECs were optimized to reproduce NN scattering data, while the $\pi \mathrm{N}$ LECs were fixed. The resulting narrow Gaussian distribution of the NN LECs $\left(\vec{a}_{\mathrm{NN}}\right)$ is shown in Fig. 2 of Ref. [6] while the mean values of both NN and $\pi \mathrm{N}$ LECs - here denoted $\vec{a}_{\mathrm{NN}}^{*}$ and $\vec{a}_{\pi \mathrm{N}}^{*}$, respectively—are listed in Appendix B of the same paper. In the present analysis we will use the fixed parameters, $\vec{a}_{\mathrm{NN}}^{*}$ and $\vec{a}_{\pi \mathrm{N}}^{*}$, when propagating the uncertainties associated with $3 \mathrm{~N}$ LECs.

The output from the parameter estimation performed in Ref. [6] was a multidimensional posterior PDF that was sampled using Markov chain Monte Carlo (MCMC) methods. Such parameter posteriors can be used to identify correlations between LECs and to propagate uncertainties to nuclear observables.

Marginalizing the the posterior $\operatorname{pr}\left(\vec{a}, \bar{c}^{2}, Q \mid \mathcal{D}, I\right)$ of Ref. [6] over all parameters except $c_{D}$ and $c_{E}$ we obtain the PDF

$$
\operatorname{pr}_{\text {full }}\left(c_{D}, c_{E}\right)=\int d \vec{a}_{\mathrm{NN}} d \bar{c}^{2} d Q \operatorname{pr}\left(\vec{a}, \bar{c}^{2}, Q \mid \mathcal{D}, I\right),
$$

shown in Fig. 1(a).
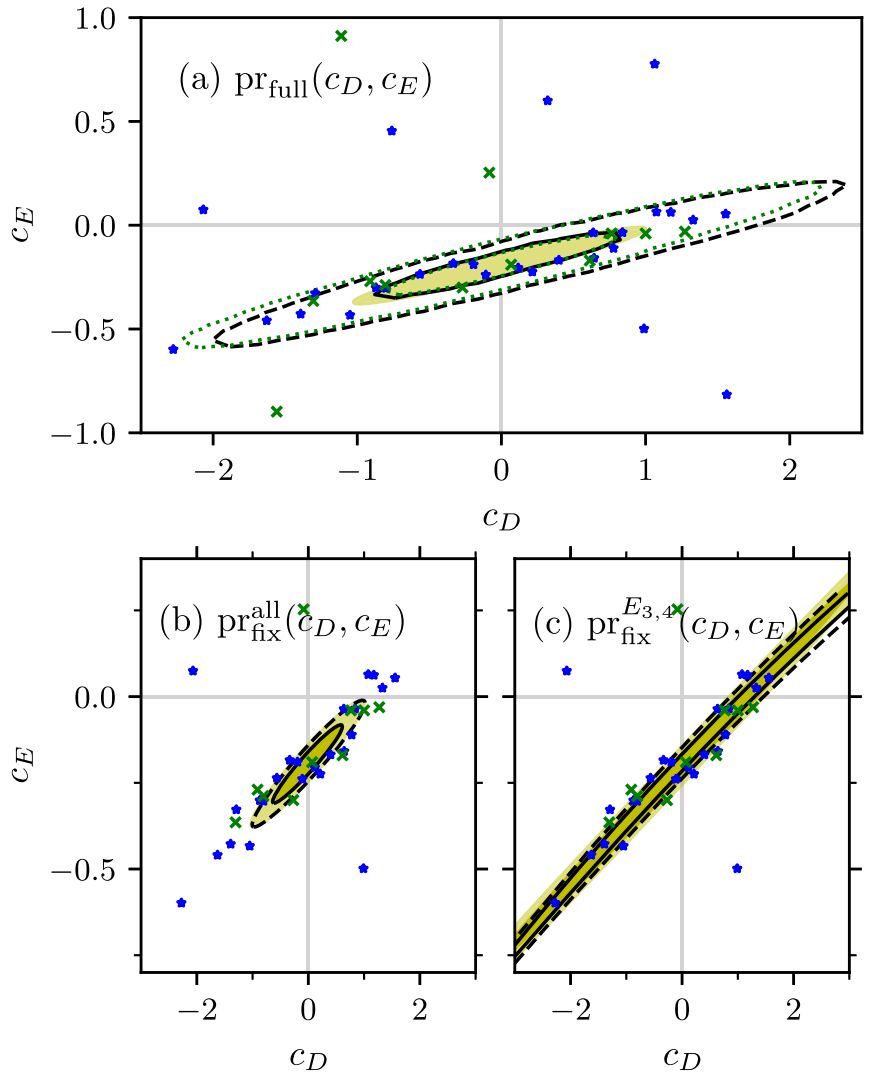

FIG. 1. Posterior pdf for $c_{D}$ and $c_{E}$ from Wesolowski et al. [6]. The main results in this work are obtained with the samples from the full posterior shown in panel (a). For comparison we also made predictions with a posterior where NN LECs and truncation error parameters were kept fixed, panels (b) and (c). The posterior shown in panel (c) was obtained with ${ }^{3} \mathrm{H}$ and ${ }^{4} \mathrm{He}$ binding energies as the only calibration data, and reveals an effective constraint in a single direction. The solid (dashed) isoprobability level encloses $68 \%$ (95\%) probability mass. The yellow dark (light) ellipses correspond to Gaussian approximations of the sampled PDFs and show the $68 \%$ $(95 \%)$ probability mass region. Note that the Gaussian approximation is too narrow to describe the full posterior in panel (a). Instead, a student $t$ distribution provides a much better fit [6] as shown by the dotted level curves. Green crosses (blue stars) correspond to training (validation) points for the emulators described in Sec. III C.

The proposition $I$ is used to implicitly subsume other known information, such as the convergence pattern (1) and the natural scale of the LECs. Although Bayesian probability theory only deals with conditional probabilities we sometimes suppress this notation in favor of notational convenience.

We will also consider two alternative parameter estimation analyses that were performed in Ref. [6]: The first one, denoted $\mathrm{pr}_{\text {fix }}^{\text {all }}\left(c_{D}, c_{E}\right)$, is shown in Fig. 1(b) and involved fixing $\bar{c}$, $Q$, and $\vec{a}_{\mathrm{NN}}$ during sampling of $c_{D}$ and $c_{E}$. It results in a more narrow distribution for $c_{D}$ and $c_{E}$. The second one, denoted $\mathrm{pr}_{\text {fix }}^{E_{3,4}}\left(c_{D}, c_{E}\right)$, is shown in Fig. 1(c) and was obtained with a much reduced set of calibration data, using only the binding energies of ${ }^{3} \mathrm{H}$ and ${ }^{4} \mathrm{He}$. It is obvious, from visual inspection of the PDF in Fig. 1(c), that these two observables are strongly correlated such that they do not provide two independent 
constraints on $c_{D}$ and $c_{E}$. Also here, $\bar{c}, Q$, and $\vec{a}_{\mathrm{NN}}$ were fixed during sampling. In this work we will use $2.5 \times 10^{6}$ $\left(c_{D}, c_{E}\right)$ samples from $\mathrm{pr}_{\text {full }}\left(c_{D}, c_{E}\right)$ to make predictions for the $A=6$ level scheme. For comparison we will also make predictions with the other two parameter PDFs for which we use $1.0 \times 10^{6}$ samples from the Gaussian approximations shown in Figs. 1(b) and 1(c), respectively.

\section{MANY-BODY CALCULATIONS}

It is a specific goal of this work to solve the many-body Schrödinger equation (MBSE)

$$
H(\vec{a})|\Psi\rangle=E(\vec{a})|\Psi\rangle
$$

for different target nuclei and with several realistic interaction parametrizations constructed for various truncations (up to N2LO) of the $\chi$ EFT expansion. As indicated, each specific many-body Hamiltonian depends on a vector of LECs $\vec{a}$.

The problem of solving Eq. (3) becomes significantly more challenging at N2LO with the inclusion of 3NFs. The $3 \mathrm{NF}$ part of our Hamiltonian is parametrized by $c_{D}$ and $c_{E}$, whose values are given by inferred probability distributions $\operatorname{pr}\left(c_{D}, c_{E}\right)$ as described in Sec. II.

Specifically, the N2LO Hamiltonian consists of the intrinsic kinetic energy plus $2 \mathrm{NF}$ and $3 \mathrm{NF}$ terms,

$$
H\left(c_{D}, c_{E}\right)=T_{\mathrm{int}}+V_{2 \mathrm{NF}}+V_{3 \mathrm{NF}}\left(c_{D}, c_{E}\right),
$$

with

$$
V_{3 \mathrm{NF}}\left(c_{D}, c_{E}\right)=V_{3 \mathrm{NF}, 2 \pi}+c_{D} V_{3 \mathrm{NF}, 1 \pi-\mathrm{ct}}+c_{E} V_{3 \mathrm{NF}, \mathrm{ct}},
$$

where the three terms in the $3 \mathrm{NF}$ correspond to twopion exchange $(3 \mathrm{NF}, 2 \pi)$, one-pion exchange plus contact $(3 \mathrm{NF}, 1 \pi-\mathrm{ct})$, and three-nucleon contact (3NF, ct) diagrams. Note that the $V_{3 \mathrm{NF}, 2 \pi}$ term is completely determined by the fixed LEC values in $\vec{a}_{\pi \mathrm{N}}$. For future reference we group all terms that will remain fixed in the Hamiltonian (4) into a constant operator $H_{0}$, while the $c_{D}$ and $c_{E}$ dependence enter linearly with operators $H_{1}$ and $H_{2}$. That is, the full Hamiltonian is written

$$
H\left(c_{D}, c_{E}\right)=H_{0}+c_{D} H_{1}+c_{E} H_{2},
$$

with

$$
\begin{aligned}
& H_{0}=T_{\mathrm{int}}+V_{2 \mathrm{NF}}+V_{3 \mathrm{NF}, 2 \pi}, \\
& H_{1}=V_{3 \mathrm{NF}, 1 \pi-\mathrm{ct}}, \\
& H_{2}=V_{3 \mathrm{NF}, \mathrm{ct}} .
\end{aligned}
$$

\section{A. JUPITERNCSM code}

To solve the MBSE (3) we use the $M$-scheme NCSM method in which the eigenstates are expanded in a many-body harmonic oscillator (HO) basis truncated on the total $\mathrm{HO}$ excitation number. Introducing the truncation parameter $N_{\max }$ we have the constraint

$$
\sum_{i=1}^{A}\left(2 n_{i}+l_{i}\right)-N_{\min } \leqslant N_{\max },
$$

where $n_{i}\left(l_{i}\right)$ is the principal HO (orbital angular momentum) quantum number of nucleon $i$ and $N_{\min }$ is the minimum total excitation number $\left(N_{\min }=2\right.$ for ${ }^{6} \mathrm{He}$ and $\left.{ }^{6} \mathrm{Li}\right)$. In this basis, the MBSE becomes a finite matrix eigenvalue problem, which is then solved iteratively using the Lanczos algorithm.

The NCSM calculations in this work were performed with the in-house developed JUPITERNCSM code [11] (unless otherwise stated). JUPITERNCSM is designed to avoid storing the full Hamiltonian matrix - a feature which makes it possible to run the code on a single compute node. Instead, the nonzero elements are generated on the fly as needed in the matrix-vector multiplication that lies at the heart of the Lanczos algorithm. This multiplication is done efficiently by precomputing the matrix representations of the $2 \mathrm{NF}$ and $3 \mathrm{NF}$ interaction operators, as well as (interaction-independent) index lists that represent nonzero transition density elements between the many-body basis states.

JUPITERNCSM employs the proton-neutron formalismbeing inspired by the code ANTOINE by Caurier et al. $[17,18]$ - with basis states being products of Slater determinants (SDs), one for each nucleon species. The basis dimensions of the $Z$-proton and $N$-neutron subspaces are much smaller than the total dimension of the combined $A$ body basis. This property allows us to create compact index lists with one-, two-, and three-nucleon transitions within the respective subspaces such that allowed transitions in the many-body basis can be reconstructed. The subspace bases are furthermore organized in blocks of fixed energy, parity, and total spin projection.

In this work we solve the MBSE (3) with full 3NF (4) for many different values of $c_{D}$ and $c_{E}$. However, much of the precomputed data are interaction independent and can be generated once for each nucleus and model space and then reused. In particular, this applies to the generation of transition index lists and to the transformation of the Hamiltonian terms $H_{0}, H_{1}$, and $H_{2}$ from the $J$ to $M$ scheme. We restrict the calculations to model spaces with $N_{\max } \leqslant 10$ for ${ }^{6} \mathrm{Li}$. In this case the generation of index lists takes $48 \mathrm{~h}$, the $J$ to $M$ scheme transformation takes $16 \mathrm{~h}$, and each converged Lanczos diagonalization (100 iterations) takes $\lesssim 25 \mathrm{~h}$ using a single compute node with two Intel Xeon Gold 6130 having $16 \mathrm{CPU}$ cores each and $384 \mathrm{GiB}$ RAM memory. The ${ }^{6} \mathrm{He}$ calculations could only be performed up to $N_{\max }=8$ due to large memory consumption when computing the three-neutron transitions.

When running JUPITERNCSM we have the choice between two different stopping criteria for the Lanczos algorithm. Option (1) terminates the algorithm when the difference between two subsequent iterations of the desired eigenvalue is less than a specified tolerance $\epsilon_{1}$. Option (2) terminates when the difference between two eigenvectors from subsequent Lanczos iterations (measured as the 2-norm) reaches below a specific tolerance $\epsilon_{2}$. This second option was used in this study when seeking high-precision eigenvectors for the construction of EC emulators (see Sec. III C).

\section{B. Exact diagonalization results}

The NCSM convergence for ${ }^{4,6} \mathrm{He}$ and ${ }^{6} \mathrm{Li}$ is shown in Fig. 2 as a function of the basis frequency, and in Fig. 3 as a function of basis truncation $N_{\max }$ for fixed basis frequency $\hbar \Omega=20 \mathrm{MeV}$. In all calculations we use a fixed NN force as 

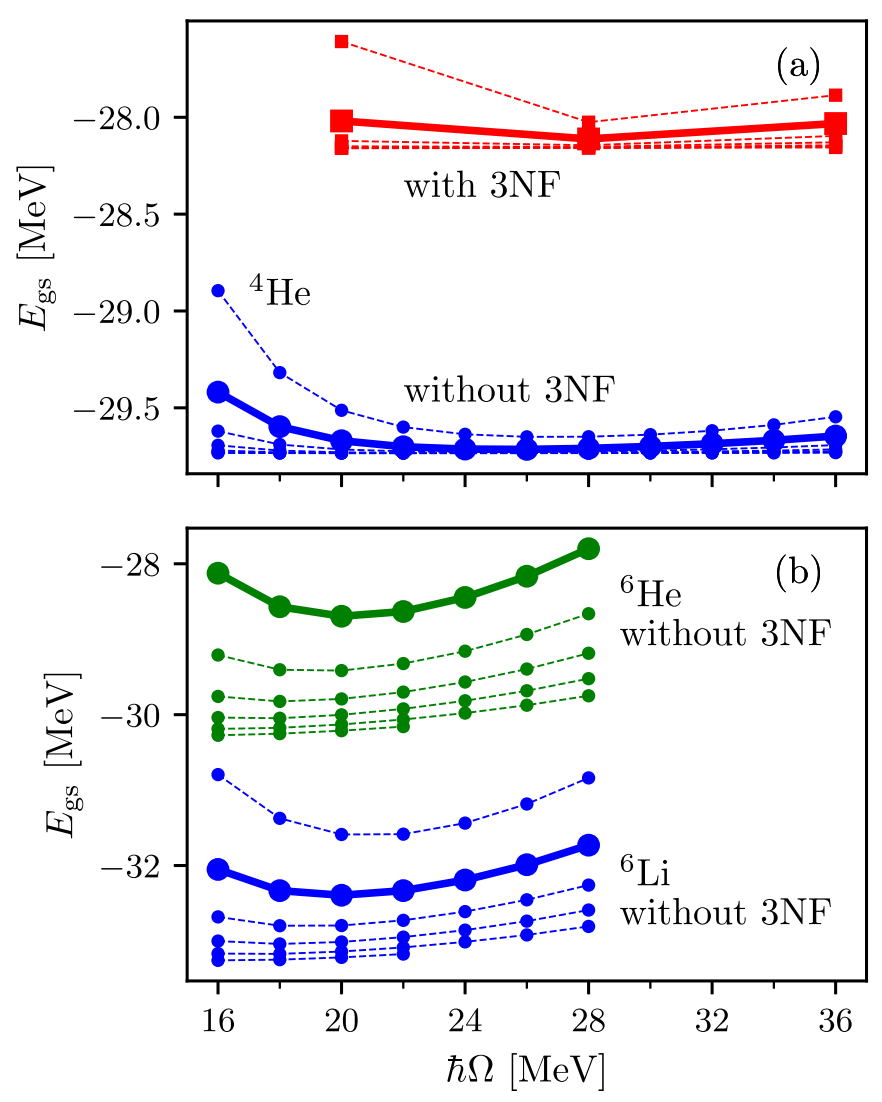

FIG. 2. NCSM convergence as a function of the basis frequency $\hbar \Omega$ for (a) ${ }^{4} \mathrm{He}$ and (b) ${ }^{6} \mathrm{Li},{ }^{6} \mathrm{He}$ computed with the N2LO Hamiltonian without $3 \mathrm{NF}$. For ${ }^{4} \mathrm{He}$ we show also results with a fixed $3 \mathrm{NF}$ $\left(c_{D}=-0.03, c_{E}=-0.20\right)$. The lines connect results at fixed basis truncation, with variationally decreasing energies from $N_{\max }=8$ to $N_{\max }=20(18)$ for $A=4(6)$. The thick solid lines correspond to $N_{\max }=10(8)$ which is the largest truncation used in the construction of emulators with $3 \mathrm{NFs}$ for ${ }^{4} \mathrm{He}$ and ${ }^{6} \mathrm{Li}\left({ }^{6} \mathrm{He}\right)$. These results show that the variational minimum appears near $\hbar \Omega=20 \mathrm{MeV}$ for all isotopes.

described in Sec. II. For ${ }^{4} \mathrm{He}$ we also show results including the $3 \mathrm{NF}$ with a maximum a posteriori (MAP) point estimate for the parameters $c_{D}=-0.03$ and $c_{E}=-0.20$ obtained from $\operatorname{pr}_{\text {full }}\left(c_{D}, c_{E}\right)$.

The thick solid lines in Fig. 2 correspond to the largest truncation used with the JUPITERNCSM code, namely $N_{\max }=10$ for ${ }^{4} \mathrm{He}$ and ${ }^{6} \mathrm{Li}$, and $N_{\max }=8$ for ${ }^{6} \mathrm{He}$. Up to this truncation we have been able to validate the ground-state energy results from different NCSM codes: NSOPT [19] (for $A=4$ ), PANTOINE [20] (for $A=4,6$; with 2NF only) and JUPITERNCSM (all nuclei; including full $3 \mathrm{NF}$ ). In addition, results for $A=$ 4,6 nuclei using the $\mathrm{N}_{2} \mathrm{LO}_{\text {sat }}$ interaction [21] (with full 3NF) have been validated by finding $\approx 1 \mathrm{keV}$ differences in groundstate energies from JUPITERNCSM and the no-core shell-model Slater determinant (NCSD) code [22].

We conclude from these convergence studies that $\hbar \Omega=$ $20 \mathrm{MeV}$ is the optimal basis frequency to use for this range of isotopes with these interactions. This basis frequency will therefore be used for the construction of emulators.
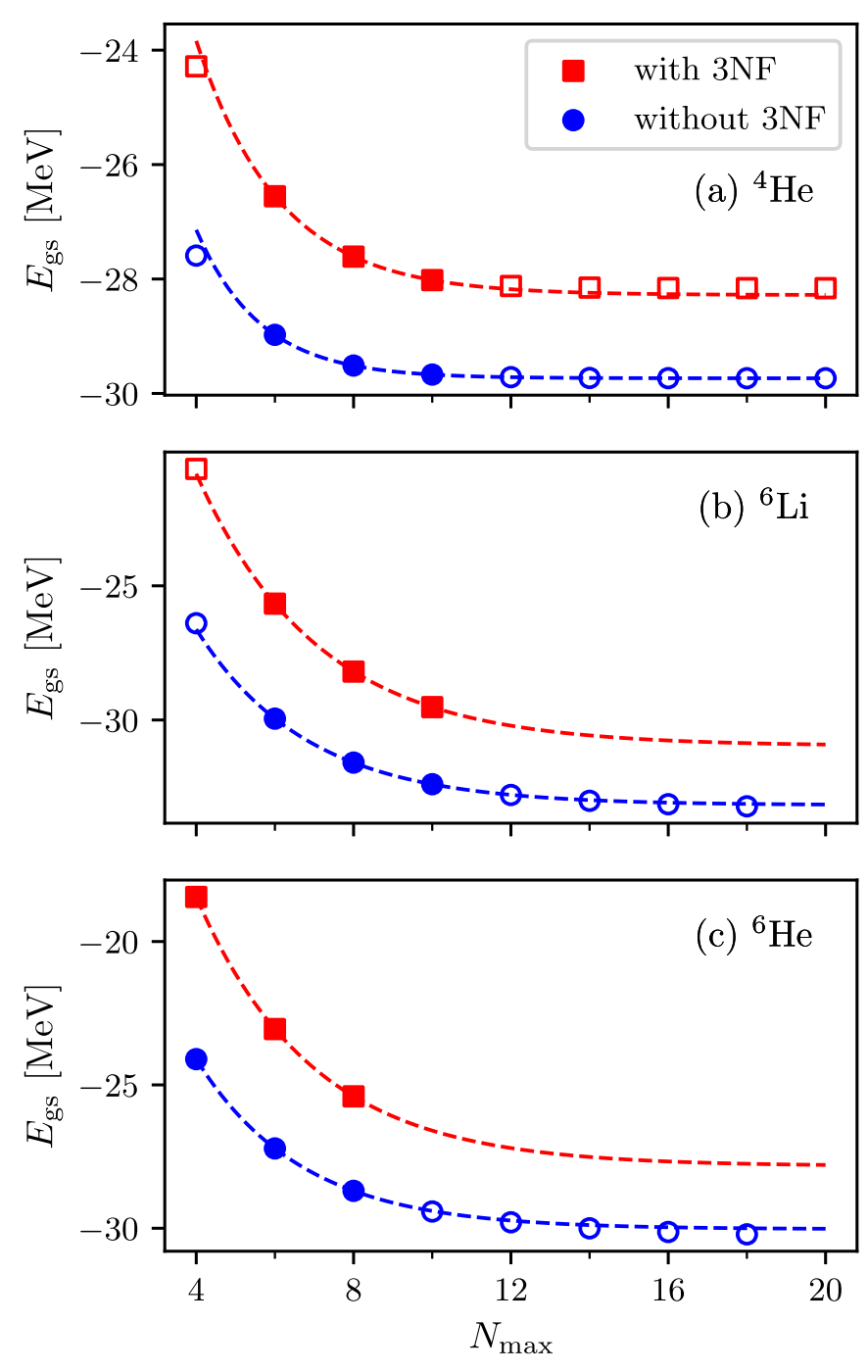

FIG. 3. NCSM convergence as a function of the basis truncation $N_{\max }$ with a fixed basis frequency $\hbar \Omega=20 \mathrm{MeV}$. Results are shown for (a) ${ }^{4} \mathrm{He}$, (b) ${ }^{6} \mathrm{Li}$, and (c) ${ }^{6} \mathrm{He}$ computed with the N2LO Hamiltonian with (square markers) and without (round markers) the $3 \mathrm{NF}$. Convergence is slower with the $3 \mathrm{NF}$ as also indicated by the exponential extrapolation curves (dashed lines). The exponential fits are made with data from $N_{\max }=6,8,10$ for ${ }^{4} \mathrm{He}$ and ${ }^{6} \mathrm{Li}$ and 4, 6, 8 for ${ }^{6} \mathrm{He}$ as shown by the filled markers.

The fact that we use the ab initio NCSM implies that we make controlled approximations when solving the many-body problem. This provides an opportunity to quantify the magnitude of our method errors, which is a prerequisite when aiming for predictive power. We express the fully converged prediction from the many-body problem as

$$
y_{\mathrm{NCSM}, \infty}(\vec{a})=y_{\mathrm{NCSM}}(\vec{a})+\delta y_{\mathrm{NCSM}}(\vec{a}),
$$

where $y_{\mathrm{NCSM}}$ is the result of our (basis truncated) ab initio many-body solver and $\delta y_{\mathrm{NCSM}}$ is the corresponding method error. In this work we will adopt a statistical model for this error term. The convergence formula that forms the foundation for our error model is presented in the following, while further details will be given in Sec. IV B. 
TABLE I. Results at LO, NLO, and N2LO for the $A=4,6$ binding energies. For LO and NLO, these correspond to the MLE from [6]. The N2LO computations use $\pi \mathrm{N}$ and NN LECs from [6,15] and the MAP point estimate is obtained with $c_{D}=-0.03$ and $c_{E}=-0.20$. The variational minimum obtained in the largest model space is shown in the $E_{\mathrm{NCSM}}$ column for each order, while the extrapolated result (10) with error estimates are shown in the $E_{\mathrm{NCSM}}+\mu_{\delta E}$ column, where $\mu_{\delta E} \equiv \mathbb{E}\left[\delta y_{\mathrm{NCSM}}\right]$. Experimental data are from [26,27] and have uncertainties that are negligible in this context.

\begin{tabular}{|c|c|c|c|c|c|c|c|}
\hline & \multicolumn{2}{|c|}{$\mathrm{LO}_{\mathrm{MLE}}$} & \multicolumn{2}{|c|}{$\mathrm{NLO}_{\mathrm{MLE}}$} & \multicolumn{2}{|c|}{$\mathrm{N} 2 \mathrm{LO}_{\mathrm{MAP}}$} & Experiment \\
\hline$E\left({ }^{4} \mathrm{He}\right)[\mathrm{MeV}]$ & -24.08 & -24.09 & -30.21 & -30.21 & -28.16 & -28.16 & $-28.296[26]$ \\
\hline$E\left({ }^{6} \mathrm{He}\right)[\mathrm{MeV}]$ & -19.23 & -20.34 & -28.49 & -28.79 & -25.40 & -28.16 & $-29.271[27]$ \\
\hline$E\left({ }^{6} \mathrm{Li}\right)[\mathrm{MeV}]$ & -19.83 & -21.32 & -31.54 & -31.78 & -29.52 & -31.13 & $-31.994[27]$ \\
\hline
\end{tabular}

In the case of HO basis expansion methods, such as the NCSM, it has been shown that analyses in terms of ultraviolet and infrared length scales offer much insight into errors resulting from finite basis truncations [20,23-25]. In this work, however, we restrict ourselves to a simpler analysis of the convergence using an exponential form

$$
E\left(N_{\max }\right)=E_{\infty}+a \exp \left(-b N_{\max }\right),
$$

which is motivated for infrared extrapolations at large $N_{\max }$ with fixed ultraviolet scale [20]. In practice, this functional form is fitted at a fixed frequency and with the parameter $E_{\infty}$ being an estimator of the converged result $y_{\mathrm{NCSM}, \infty}$. We note that the convergence distance

$$
\Delta E_{\infty}\left(N_{\max }\right) \equiv E_{\infty}-E\left(N_{\max }\right)
$$

must be negative since the NCSM is a variational method. We will return to this extrapolation formula in Sec. IV B when we introduce the statistical model for the method error.

The convergence of the ground-state energy as a function of the basis truncation is shown in Fig. 3. The validation results at large $N_{\max }$ with $2 \mathrm{NF}$-only interactions indicate that the exponential extrapolation slightly underestimates the convergence distance for $A=6$ nuclei. We will later incorporate this finding into our model of the method error. Furthermore, the observed convergence with respect to $N_{\max }$ with a full Hamiltonian including $3 \mathrm{NFs}$ is somewhat slower than the one with $2 \mathrm{NF}$ only.

We have used the LEC values from Wesolowski et al. [6] to study the order-by-order convergence of $A=4,6$ binding energies. These point estimates correspond to a maximum likelihood estimation (MLE) for the LO and NLO Hamiltonians, and a MAP for the N2LO Hamiltonian. The results from our NCSM exact diagonalizations are shown in Table I. Here we also show extrapolated results with inclusion of the estimated mean method error $\mu_{\delta E}$; see Sec. IV B 1 . We note in particular that our LO estimate corresponds to underbinding of these systems, which is in stark contrast with many other studies that give strong overbinding at LO $[8,16]$. This difference can be traced to the fit to low-energy scattering data in [6] where the $\chi$ EFT expansion parameter $Q=m_{\pi} / \Lambda_{b}$ was employed in that domain — with $m_{\pi}$ the pion mass and $\Lambda_{b}$ the $\chi$ EFT breakdown scale. As a result, the deuteron energy is $E_{d}=-0.6 \mathrm{MeV}$ at $\mathrm{LO}$, and this underbinding prevails for the $A=4,6$ systems.

\section{Eigenvector continuation emulators}

The specific aim in this work is the computation of a PPD for the ground-state energies of several many-body systems. This requires the ability to solve the MBSE repeatedly for different nuclear systems and for many samples from the Hamiltonian parameter PDF. In this work we will solve for ${ }^{4} \mathrm{He},{ }^{6} \mathrm{Li}$, and ${ }^{6} \mathrm{He}$ with $\approx 2.5 \times 10^{6}$ samples from $\operatorname{pr}_{\text {full }}\left(c_{D}, c_{E}\right)$. This is achieved by exploiting the method of EC $[12,13,28]$ to mimic the solution of the full problem with high accuracy at a fraction of the computational cost.

Given a Hermitian matrix that depends smoothly on some continuous parameters - in our case $H\left(c_{D}, c_{E}\right)$ - this method can be used to construct an emulator that performs very well in a large parameter domain. The training of such an emulator requires a small set of training vectors obtained by solving the full problem for a corresponding set of training points in $\left(c_{D}, c_{E}\right)$ space.

For each nucleus $\left({ }^{4,6} \mathrm{He},{ }^{6} \mathrm{Li}\right)$ and model space $\left(N_{\max } \in\right.$ $\{4,6,8,10\})$, we compute the ground state by solving Eq. (3) using JUPITERNCSM for a total of 16 training points $\left\{c_{D, k}, c_{E, k}\right\}_{k=1}^{16}$. Eight of these training points correspond to random draws from the posterior $\operatorname{pr}_{\text {full }}\left(c_{D}, c_{E}\right)$ that is shown in Fig. 1(a), while the remaining eight were drawn from a large square: $c_{D}, c_{E} \sim \mathcal{U}(-2.5,2.5)$. After performing diagonalizations - using the eigenvector convergence criterion with $\epsilon_{2}=10^{-6}$ - this results in 16 training vectors for each nucleus and model space, i.e.,

$$
\left|\Phi_{k}\right\rangle=\left|\Psi\left(c_{D, k}, c_{E, k}\right)\right\rangle, \quad k=1,2, \ldots, 16 .
$$

When using EC, the Hamiltonian is projected onto the subspace that is spanned by the training vectors. We denote our subspace-projected Hamiltonian as $M\left(c_{D}, c_{E}\right)$ and its matrix elements are computed as

$$
\begin{aligned}
\left(M\left(c_{D}, c_{E}\right)\right)_{i, j}= & \left\langle\Phi_{i}\left|H_{0}\right| \Phi_{j}\right\rangle+c_{D}\left\langle\Phi_{i}\left|H_{1}\right| \Phi_{j}\right\rangle \\
& +c_{E}\left\langle\Phi_{i}\left|H_{2}\right| \Phi_{j}\right\rangle \\
= & \left(M_{0}\right)_{i, j}+c_{D}\left(M_{1}\right)_{i, j}+c_{E}\left(M_{2}\right)_{i, j},
\end{aligned}
$$

where we note that $M_{0}, M_{1}$, and $M_{2}$ are $16 \times 16$ matrices that can be computed once per nucleus and model space.

There is no guarantee that the training vectors are orthogonal. Therefore, we also construct the norm matrix $N$ such that

$$
(N)_{i, j}=\left\langle\Phi_{i} \mid \Phi_{j}\right\rangle
$$


The subspace-projected MBSE becomes a generalized eigenvalue problem

$$
M\left(c_{D}, c_{E}\right) \vec{v}\left(c_{D}, c_{E}\right)=\lambda\left(c_{D}, c_{E}\right) N \vec{v}\left(c_{D}, c_{E}\right),
$$

where $\lambda(\vec{v})$ is the eigenvalue (eigenvector). Since we are projecting the NCSM Hamiltonian onto a subspace, the EC method is variational and therefore $E_{\mathrm{gs}}^{\mathrm{EC}}=\min \left\{\lambda\left(c_{D}, c_{E}\right)\right\} \geqslant$ $E_{\mathrm{gs}}\left(c_{D}, c_{E}\right)$. It turns out that $E_{\mathrm{gs}}^{\mathrm{EC}}$ is a very good approximation of the NCSM ground-state energy.

A validation of the $\mathrm{EC}$ emulators is performed by selecting an additional $40\left(c_{D}, c_{E}\right)$ samples, 20 of which are new draws from the posterior $\operatorname{pr}_{\text {full }}\left(c_{D}, c_{E}\right)$, and 20 from the large square: $c_{D}, c_{E} \sim \mathcal{U}(-2.5,2.5)$. We then compare emulated groundstate energies $E_{\mathrm{gs}}^{\mathrm{EC}}$, obtained by solving Eq. (15), and full numerical solutions $E_{\mathrm{gs}}$ obtained with JUPITERNCSM using an eigenvalue convergence criterion with $\epsilon_{1}=10^{-7}$. The results of this validation are shown in Fig. 4 for the largest $N_{\max }$ used for each nucleus. Here we focus on the most interesting region in parameter space and include the 20 validation points (blue stars) and eight training points (green crosses) that are drawn from the posterior distribution (see Fig. 1).

As can be seen, the relative difference of the validation points is very small for all three nuclei. For the two helium isotopes this difference is around $10^{-9}$, while for ${ }^{6} \mathrm{Li}$ it is around $10^{-7}$, which is close to the convergence criterion that we used for the Lanczos algorithm in JUPITERNCSM. Therefore, we conclude that EC-emulated ground-state energies are basically as accurate as the same values computed in the full Hilbert space using JUPITERNCSM for $c_{D}, c_{E}$ values within the posterior $\mathrm{pr}_{\text {full }}\left(c_{D}, c_{E}\right)$. In fact, we find almost the same precision in the large square of $\left(c_{D}, c_{E}\right)$ parameter values, except for points that produce extreme energies $\left(E_{\mathrm{gs}}<-100 \mathrm{MeV}\right)$ for which the relative difference becomes $\approx 10^{-2}$.

\section{POSTERIOR PREDICTIVE DISTRIBUTIONS}

In this section we will study and draw samples from the PPD, which we for the NCSM predictions define as the marginalized joint distribution

$$
\begin{aligned}
\operatorname{pr}\left(\boldsymbol{y}_{\mathrm{NCSM}} \mid \mathcal{D}, I\right) & =\int d \vec{a} \operatorname{pr}\left(\boldsymbol{y}_{\mathrm{NCSM}}, \vec{a} \mid \mathcal{D}, I\right) \\
& =\int d \vec{a} \operatorname{pr}\left(\boldsymbol{y}_{\mathrm{NCSM}} \mid \vec{a}, \mathcal{D}, I\right) \operatorname{pr}(\vec{a} \mid \mathcal{D}, I),
\end{aligned}
$$

where in the final step we used the product rule of probability. To draw $S$ samples from this PPD we straightforwardly evaluate $\boldsymbol{y}_{\mathrm{NCSM}}(\vec{a})$ for $S$ samples of $\vec{a}$ drawn from the posterior PDF $\operatorname{pr}(\vec{a} \mid \mathcal{D}, I)$ for the LECs. This procedure is succinctly described by the set definition

$$
\operatorname{PPD}_{\mathrm{NCSM}}=\left\{\boldsymbol{y}_{\mathrm{NCSM}}(\vec{a}): \vec{a} \sim \operatorname{pr}(\vec{a} \mid \mathcal{D}, I)\right\},
$$

i.e., the set of all model predictions computed over likely values of the LECs. In our specific case, with fixed NN and $\pi \mathrm{N} \vec{a}$, the posterior PDF corresponds to

$$
\operatorname{pr}(\vec{a} \mid \mathcal{D}, I)=\delta\left(\vec{a}_{\mathrm{NN}}-\vec{a}_{\mathrm{NN}}^{*}\right) \delta\left(\vec{a}_{\pi \mathrm{N}}-\vec{a}_{\pi \mathrm{N}}^{*}\right) \operatorname{pr}\left(c_{D}, c_{E}\right),
$$
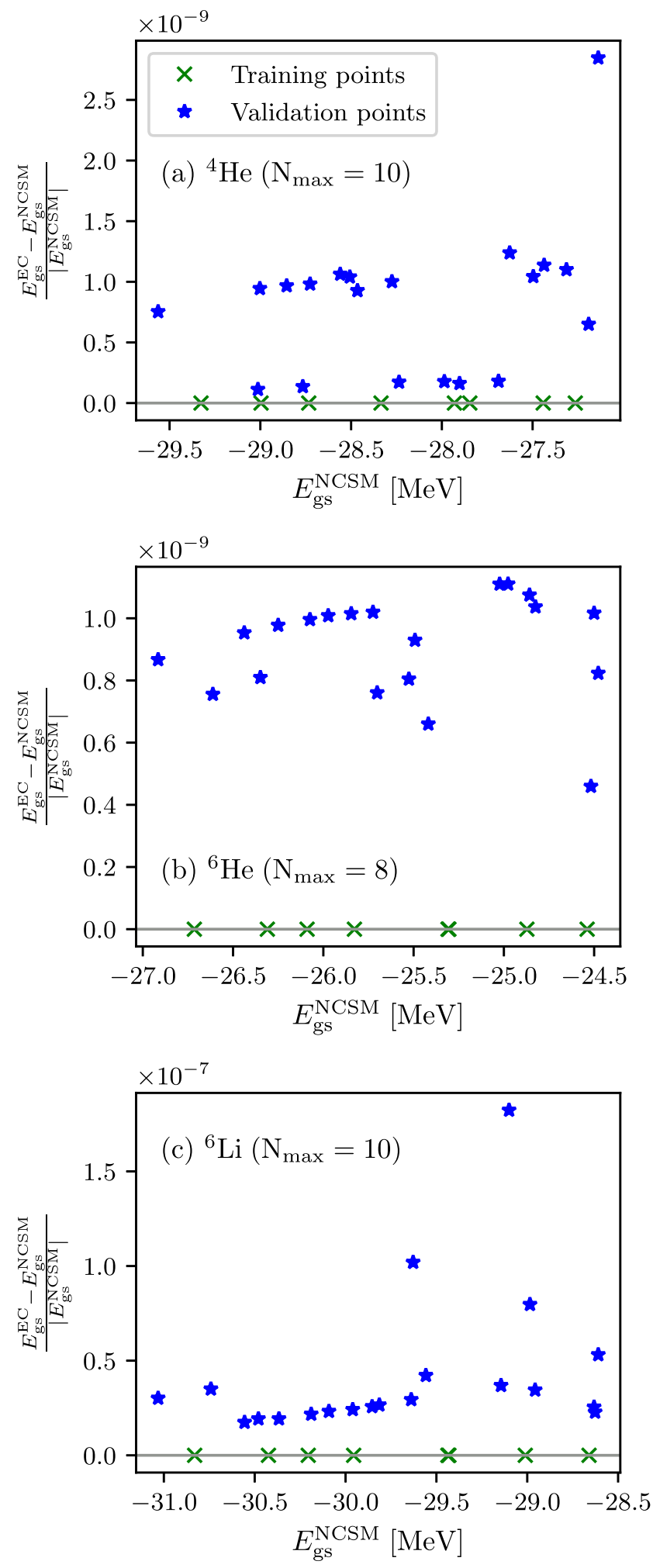

FIG. 4. Cross validation of the emulators by comparing the relative differences between the full solution of the Schrödinger equation (at fixed basis space truncation $N_{\max }$ ) and those of the EC emulators at 20 validation points (blue stars). We also demonstrate the validation at the training points (green crosses), where the subspace eigensolution should be identical to the full one. Note that the ground-state energies from the emulator are guaranteed to be an upper bound on the exact NCSM energies. 


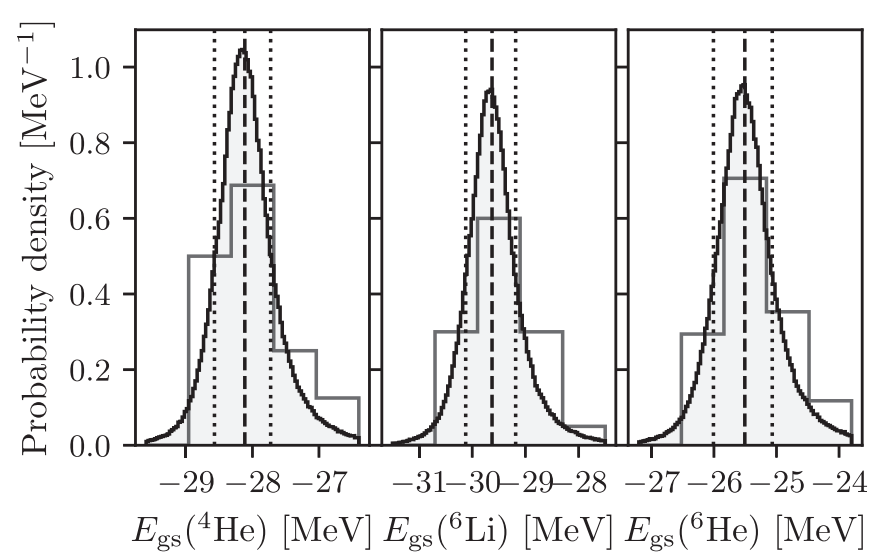

FIG. 5. Marginal distributions from $\mathrm{PPD}_{\mathrm{NCSM}}$ (17) illustrating the parametric uncertainties from the posterior distribution for $\left(c_{D}, c_{E}\right)$ (2) propagated to ground-state energies of ${ }^{4} \mathrm{He},{ }^{6} \mathrm{Li}$ computed with $N_{\max }=10$ and ${ }^{6} \mathrm{He}$ with $N_{\max }=8$. The median value and the $68 \%$ Bayesian credible interval is indicated by dashed and dotted lines, respectively. See also Table II. The open, grey histograms on the diagonal represent low-statistics results with only 25 samples (five bins).

with different $\operatorname{pr}\left(c_{D}, c_{E}\right)$ distributions introduced in Sec. II. Such results are shown in Figs. 5 and 6 and discussed below. They illustrate the parametric uncertainty, which only implicitly contains uncertainties that stem from the truncation of our many-body solver (method uncertainty) or the truncation of our EFT (model uncertainty). More specifically, quantified errors for calibration observables that arise from these truncations are propagated via the inference of the LEC posterior.

The explicit incorporation of the model discrepancy, method uncertainty, and emulator error terms for predicted observables is consolidated in the full PPD, which will be discussed in Sec. IV B.

\section{A. NCSM posterior predictive distribution}

The relevant question on the magnitude of parametric uncertainties in predictions made with our nuclear $a b$ initio approach is addressed with the NCSM PPD (17). For this purpose we utilize the few-body-constrained LEC posterior [6] discussed in Sec. II, together with our EC emulators for $A=4,6$ binding energies (see Sec. IIIC). These fast and accurate emulators make it possible for us to predict the ${ }^{4,6} \mathrm{He}$ and ${ }^{6} \mathrm{Li}$ binding energies for $\approx 2.5 \times 10^{6}$ samples from $\operatorname{pr}_{\text {full }}\left(c_{D}, c_{E}\right)(2)$.

The resulting marginal distributions are shown in Fig. 5 together with the medians and the $68 \%$ Bayesian credible intervals (obtained as the smallest region that contains $68 \%$ of the probability mass). Numerical values for these summary statistics are presented in Table II.

The consistency of our approach can be assessed by studying the $\mathrm{PPD}_{\mathrm{NCSM}}$ for ${ }^{4} \mathrm{He}$ since this observable was part of the calibration data for our model. Such model checking was performed already in Ref. [6] and the shape of our ${ }^{4} \mathrm{He}$ distribution in Fig. 5 compares well with the results shown in the diagonal panel (second row) of Fig. 4 in that paper. However, it should be noted that while our ${ }^{4} \mathrm{He}$ emulator was
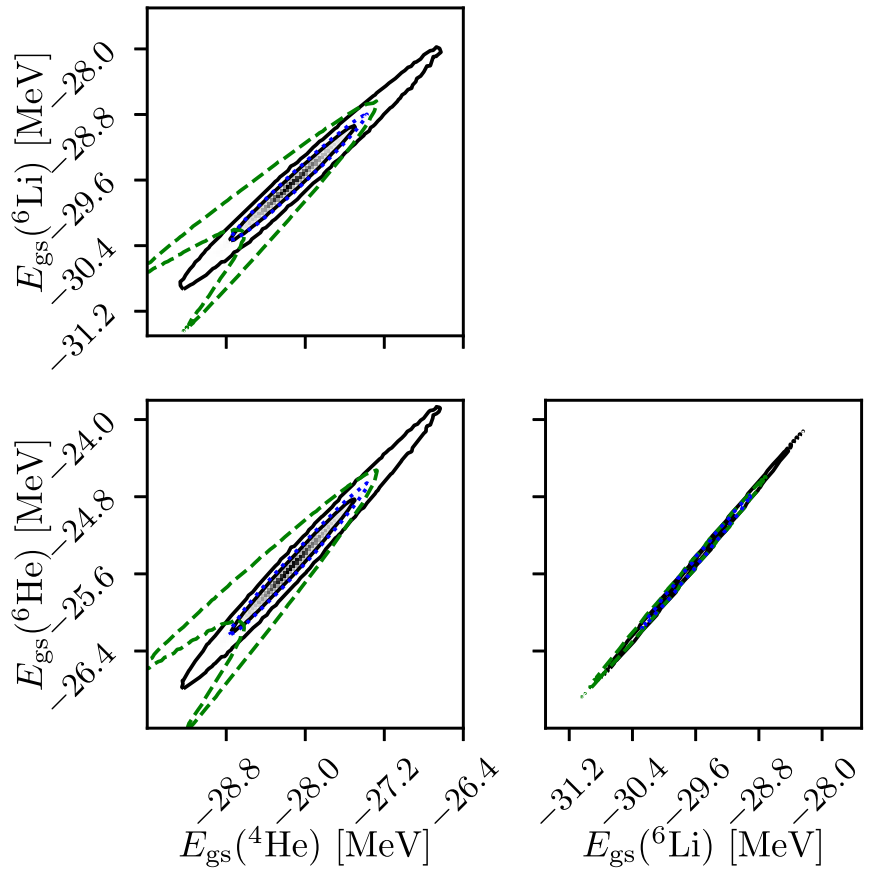

FIG. 6. Bivariate distributions from $\mathrm{PPD}_{\mathrm{NCSM}}$ (17) illustrating the parametric uncertainties from the posterior distribution $\operatorname{pr}_{\text {full }}\left(c_{D}, c_{E}\right)$ (2) propagated to ground-state energies of ${ }^{4} \mathrm{He},{ }^{6} \mathrm{Li}$ computed with $N_{\max }=10$ and ${ }^{6} \mathrm{He}$ with $N_{\max }=8$. The $68 \%$ and $95 \%$ probability mass regions are shown by the level curves. For comparison, the $95 \%$ regions for the posteriors $\operatorname{pr}_{\mathrm{fix}}^{E_{3,4}}\left(c_{D}, c_{E}\right)$ and $\operatorname{pr}_{\text {fix }}^{\text {all }}\left(c_{D}, c_{E}\right)$ are shown with green dashed and blue dotted curves, respectively.

TABLE II. Median predictions and 68\% (95\%) Bayesian credible intervals shown in the first (second) row for each observable. All values are in $\mathrm{MeV}$ and the intervals are presented as differences from the median. The NCSM PPD is obtained by using the raw output from our emulators $\left({ }^{4} \mathrm{He},{ }^{6} \mathrm{Li}\right.$ computed with $N_{\max }=$ 10 and ${ }^{6} \mathrm{He}$ with $N_{\max }=8$ ). The full PPD, however, includes also statistical models for the method and model errors (see text for details). Experimental energies are listed in Table I and result in the energy differences: $S_{d}\left({ }^{6} \mathrm{Li}\right)=1.473 \mathrm{MeV}, S_{2 n}\left({ }^{6} \mathrm{He}\right)=0.975 \mathrm{MeV}$, and $Q_{\beta^{-}}\left({ }^{6} \mathrm{He}\right)=3.505 \mathrm{MeV}$

\begin{tabular}{lccccc}
\hline \hline & \multicolumn{2}{c}{ NCSM PPD } & & \multicolumn{2}{c}{ Full PPD } \\
\cline { 2 - 3 } \cline { 6 - 6 } Observable & median & CI $(68 / 95 \%)$ & & median & CI $(68 / 95 \%)$ \\
\hline$E\left({ }^{4} \mathrm{He}\right)$ & -28.11 & {$[-0.46,+0.39]$} & -28.23 & {$[-0.80,+0.75]$} \\
& & {$[-0.99,+1.20]$} & & {$[-1.59,+1.65]$} \\
$E\left({ }^{6} \mathrm{Li}\right)$ & -29.63 & {$[-0.50,+0.44]$} & -31.34 & {$[-0.92,+0.89]$} \\
& & {$[-1.09,+1.35]$} & & {$[-1.84,+1.92]$} \\
$E\left({ }^{6} \mathrm{He}\right)$ & -25.51 & {$[-0.50,+0.44]$} & -28.42 & {$[-0.96,+0.95]$} \\
$S_{d}\left({ }^{6} \mathrm{Li}\right)$ & -0.71 & {$[-0.08,+0.08]$} & 0.89 & {$[-0.44,+0.44]$} \\
& & {$[-0.21,+0.21]$} & & {$[-0.87,+0.88]$} \\
$S_{2 n}\left({ }^{6} \mathrm{He}\right)$ & -2.61 & {$[-0.08,+0.07]$} & 0.19 & {$[-0.61,+0.58]$} \\
$Q_{\beta}\left({ }^{6} \mathrm{He}\right)$ & 4.90 & {$[-0.01,+0.00]$} & 3.71 & {$[-0.65,+0.64]$} \\
& & {$[-0.01,+0.02]$} & & {$[-1.26,+1.27]$} \\
\hline \hline
\end{tabular}


constructed with $N_{\max }=10$ in the $M$-scheme NCSM, those of Wesolowski et al. [6] were based on a Jacobi-coordinate NCSM implementation and used $N_{\max }=18$. This gap in model space results in $\approx 100 \mathrm{keV}$ energy difference.

Accurate emulators might not always be available for the observable of interest. For that reason we demonstrate a low-statistics representation of the same results by open histograms in Fig. 5. These results are based on merely 25 samples from $\mathrm{pr}_{\text {full }}\left(c_{D}, c_{E}\right)$. Both the median and the standard deviation of the predictions made with the low-statistics representation agrees to within $10 \mathrm{keV}$ with the summary statistics obtained from the full list of samples. Although small statistics might not allow a very precise quantification of credible intervals, it is clear that valuable parametric uncertainty estimates can still be extracted.

The bivariate distributions from $\mathrm{PPD}_{\mathrm{NCSM}}$ are shown in Fig. 6. We observe very strong correlations between the predicted binding energies, as could be expected. This means that the uncertainties in relative energies will be much smaller, as we will discuss in more detail below. For comparison we also show the bivariate distributions that are obtained when using samples from the alternative parameter posteriors $\operatorname{pr}_{\text {fix }}^{\text {all }}\left(c_{D}, c_{E}\right)$ and $\operatorname{pr}_{\text {fix }}^{E_{3,4}}\left(c_{D}, c_{E}\right)$; see Fig. 1 and the discussion in Sec. II. The fact that the truncation-error parameters $\bar{c}$ and $Q$ were fixed leads to a much narrower (more Gaussian) distribution of $\left(c_{D}, c_{E}\right)$ in $\operatorname{pr}_{\mathrm{fix}}^{\text {all }}\left(c_{D}, c_{E}\right)$, which is also reflected in the tighter NCSM PPD (blue, dotted region).

Even more interesting is the behavior of the NCSM PPD from $\operatorname{pr}_{\text {fix }}^{E_{3,4}}\left(c_{D}, c_{E}\right)$. As shown in Fig. 1(c), the use of highly correlated calibration observables, $E\left({ }^{3} \mathrm{H}\right)$ and $E\left({ }^{4} \mathrm{He}\right)$, gives an effective parameter constraint in a single direction which leads to a very broad range of allowed $\left(c_{D}, c_{E}\right)$ values. The propagation of that parametric uncertainty to predictions of the $A=6$ binding energies reveals if those new observables would be able to provide additional, independent constraints. We actually find that the bulk region of the NCSM PPD obtained from $\operatorname{pr}_{\text {fix }}^{E_{3,4}}\left(c_{D}, c_{E}\right)$ does largely overlap with the ones from $\operatorname{pr}_{\text {full }}\left(c_{D}, c_{E}\right)$ and $\operatorname{pr}_{\text {fix }}^{\text {all }}\left(c_{D}, c_{E}\right)$ that are informed by a larger set of few-body observables. This indicates that their propagated predictions for $A=6$ binding energies are very similar. However, the most extreme samples from $\operatorname{pr}_{\text {fix }}^{E_{3,4}}\left(c_{D}, c_{E}\right)$, i.e., $c_{D} \approx-5$ and $c_{D} \approx+5$, correspond respectively to the upper and lower "legs" of the green, dashed $95 \%$ confidence regions shown in the left column of Fig. 6.

\section{B. Full posterior predictive distribution}

As mentioned, the NCSM PPD does not reflect explicit uncertainties associated with the truncation of the many-body model space, nor with the truncation of the EFT expansion, in the prediction of observables. Let us therefore incorporate models for these additional, relevant errors into our final predictions. The full PPD is then defined, in analogy with Eq. (17), as the set evaluation of $\boldsymbol{y}(\vec{a})$ which is the sum

$$
\begin{aligned}
\boldsymbol{y}(\vec{a}) & =\boldsymbol{y}_{\mathrm{NCSM}}(\vec{a})+\delta \boldsymbol{y}_{\mathrm{NCSM}}(\vec{a})+\delta \boldsymbol{y}_{\mathrm{EFT}}(\vec{a})+\delta \boldsymbol{y}_{\mathrm{em}} \\
& \approx \boldsymbol{y}_{\mathrm{NCSM}}(\vec{a})+\delta \boldsymbol{y}_{\mathrm{NCSM}}(\vec{a})+\delta \boldsymbol{y}_{\mathrm{EFT}},
\end{aligned}
$$

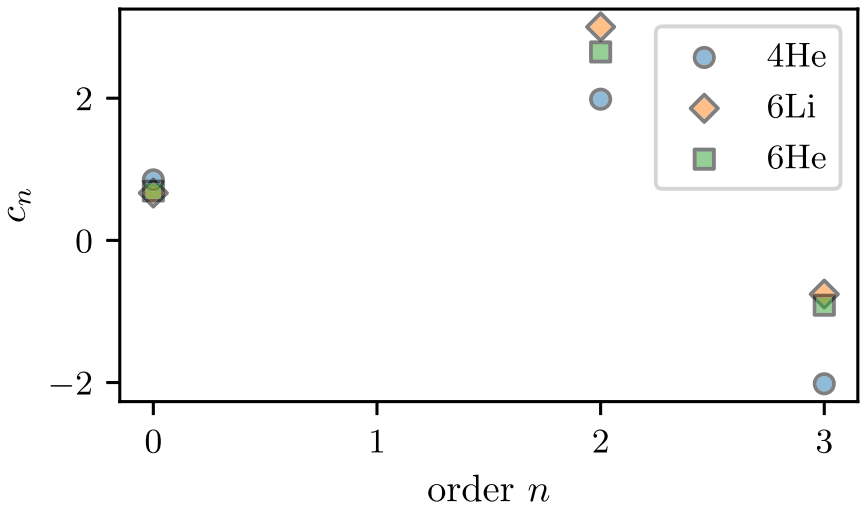

FIG. 7. Observable coefficients, $c_{n}$, for $A=4,6$ binding energies up to N2LO obtained using a chiral expansion parameter $Q=1 / 3$ for all three observables.

where it should be noted that we explicitly assume that the method error might depend on the LEC parameters while the EFT error does not. Furthermore, we assume that the emulator error is negligible compared to the other uncertainties as motivated in Sec. III C. We consider the error terms, $\delta \boldsymbol{y}_{\mathrm{NCSM}}(\vec{a})$ and $\delta \boldsymbol{y}_{\mathrm{EFT}}$, in Eq. (19) as random variables with distributions that will be discussed in detail in the following subsections.

\section{Method errors}

The method error is estimated from the observed convergence behavior and our previous experience with the NCSM using also other interaction models. In particular, we know that the NCSM is a variational approach such that results obtained at truncated model spaces represent upper bounds. This implies that the expectation value for the method error of the total energy observable $i$ is negative,

$$
\mathbb{E}\left[\delta y_{\mathrm{NCSM}, i}(\vec{a})\right] \equiv \mu_{\delta E, i}(\vec{a})<0 .
$$

Since we have constructed emulators for different model spaces, $N_{\max } \in\{4,6,8,10\}$, we can perform the extrapolation (10) for each set of LECs and extract an $\vec{a}$-dependent convergence distance $\Delta E_{\infty, i}(\vec{a})$ in Eq. (11). However, the convergence tests in Sec. III B together with previous experience [20,25,29] tells us that this simple extrapolation tends to underestimate the convergence distance by a few ten percent. To incorporate this knowledge we estimate the mean value of the method error as

$$
\begin{aligned}
\mu_{\delta E, i}(\vec{a}) & =\Delta E_{\infty, i}(\vec{a})+\sigma_{\mathrm{NCSM}, i}, \\
\text { with } \sigma_{\mathrm{NCSM}, i}(\vec{a}) & =0.2 \Delta E_{\infty, i}(\vec{a}) .
\end{aligned}
$$

The mean value for $\Delta E_{\infty}$ for the LEC samples from $\operatorname{pr}_{\text {full }}\left(c_{D}, c_{E}\right)$ is found to be $-1.46(-2.46) \mathrm{MeV}$ for ${ }^{6} \mathrm{Li}\left({ }^{6} \mathrm{He}\right)$. The standard deviation for $\Delta E_{\infty}$ is very small at 50 (120) $\mathrm{keV}$ and we therefore simplify the assignment by making $\vec{a}$-independent estimates: $\sigma_{\mathrm{NCSM}}=-290(-490) \mathrm{keV}$ for ${ }^{6} \mathrm{Li}$ $\left({ }^{6} \mathrm{He}\right)$. Furthermore, the square of this correction is used as the variance of the assigned method error.

For ${ }^{4} \mathrm{He}$ we find that the mean value for $\Delta E_{\infty}$ is $-0.26 \mathrm{MeV}$ with a very small standard deviation of 23 $\mathrm{keV}$. For this short extrapolation distance we find that the 


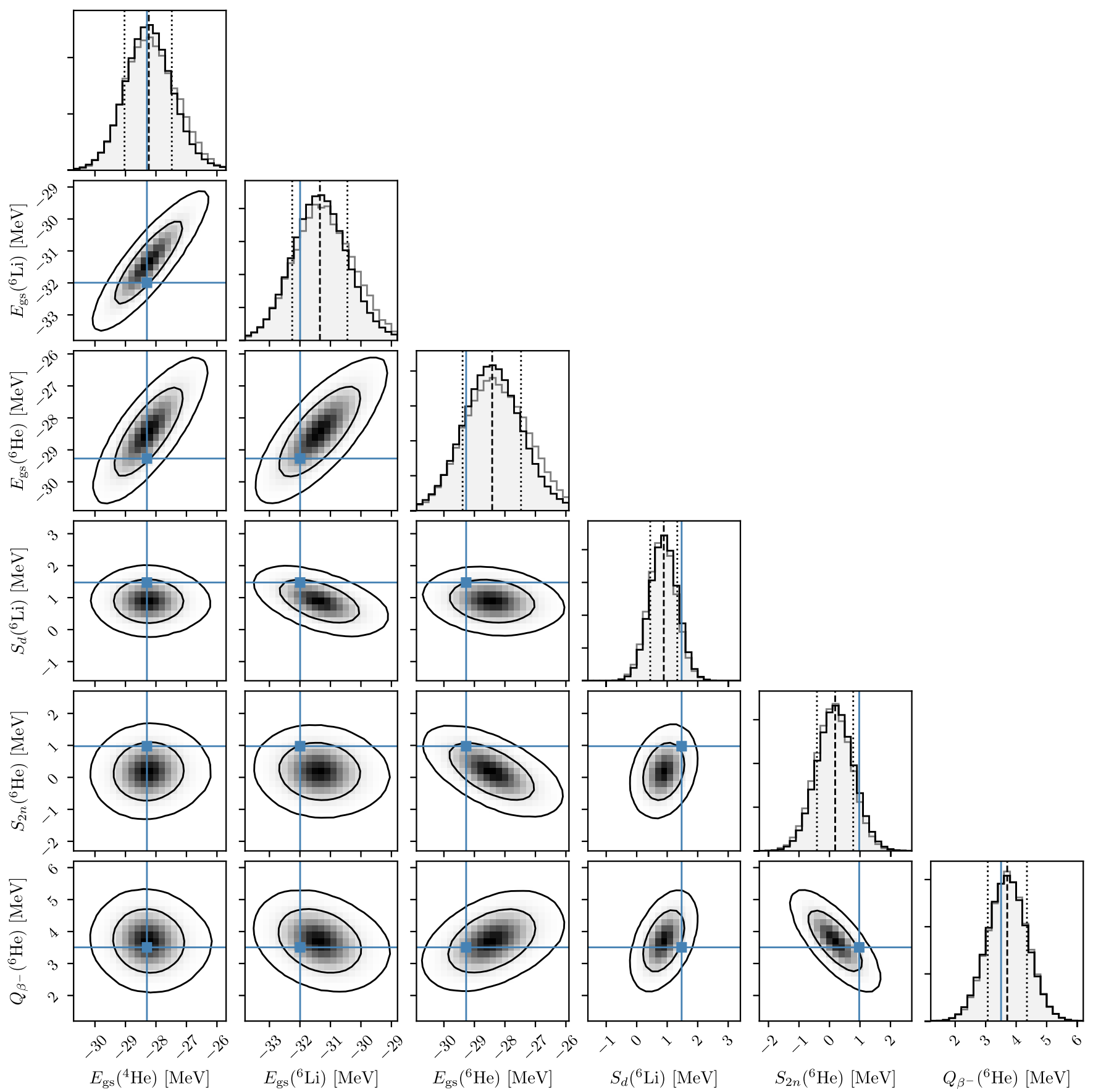

FIG. 8. Full PPD for binding energies and thresholds including both method and model (EFT truncation) uncertainties. The dashed (dotted), vertical lines on the diagonal show the median ( $68 \%$ credible interval), while the blue, solid lines indicate the experimental values. See also Table II. The open, grey histograms on the diagonal represent low-statistics results based on only 25 LEC samples (see text for details). The level curves in the off-diagonal panels show the $68 \%$ and $95 \%$ probability mass regions of the bivariate distributions.

exponential form actually overestimates the missing binding energy and gives an $E_{\infty}$ that is about $120 \mathrm{keV}$ below the converged result; see Fig. 3(a). Consequently, we assign $\sigma_{\mathrm{NCSM}}=+120 \mathrm{keV}$ for this nucleus.

In summary, we model the distribution of the method error random variables $\delta y_{\mathrm{NCSM}, i}(\vec{a})$ as normal distributions,

$$
\delta y_{\mathrm{NCSM}, i}(\vec{a}) \sim \mathcal{N}\left(\mu_{\delta E, i}(\vec{a}), \sigma_{\mathrm{NCSM}, i}^{2}\right)
$$

with an $\vec{a}$ dependence in the mean value-stemming from the observed $N_{\max }$ convergence-and a nucleus-dependent convergence uncertainty that both corrects the mean and defines the estimated variance.

\section{Model errors}

We create a statistical model for the EFT model discrepancy $\delta y_{\mathrm{EFT}}$ based on the observed terms in the assumed EFT 
convergence pattern (1). Point estimates for predictions at each order from Table I are converted to observable coefficients assuming a fixed value for the expansion parameter. Specifically we use the value, $Q=0.33$, inferred in Ref. [6] for $A=3,4$ observables since the $A=6$ systems considered in this work are clusterized and exhibit low- rather than highmomentum scales [20]. The resulting coefficients are shown in Fig. 7. It is obvious that the corrections to the three binding energies are similar at each order which indicates that the observable coefficients are strongly correlated. We will assume that these coefficients are i.i.d. random variables drawn from a single multivariate normal distribution: $\boldsymbol{c}_{\mathrm{n}} \sim \mathcal{N}(0, \Sigma)$, with the covariance matrix expressed in terms of its diagonal elements and a simple correlation matrix,

$$
\begin{aligned}
\Sigma & =\sigma R \sigma, \\
\text { where } \sigma_{i j} & = \begin{cases}\bar{c} & \text { for } i=j, \\
0 & \text { for } i \neq j,\end{cases} \\
\text { and } R_{i j} & = \begin{cases}1 & \text { for } i=j, \\
\rho & \text { for } i \neq j .\end{cases}
\end{aligned}
$$

A straightforward MLE, with the likelihood based on the data shown in Fig. 7, gives $\bar{c}=1.7$ and $\rho=0.9$. We will use those as fixed parameters in the following.

For this given model of the EFT observable coefficients, all neglected terms beyond $\mathcal{O}\left(Q^{k}\right)$ in Eq. (1) can be summed to give a distribution for the model error $\delta \boldsymbol{y}_{\mathrm{EFT}}$ [2]. Specifically, with $\boldsymbol{c}_{\mathrm{n}} \sim \mathcal{N}(0, \Sigma)$ this sum can be performed analytically $[4,5]$ and we find

$$
\begin{aligned}
\delta y_{\mathrm{EFT}} & \sim \mathcal{N}\left(0, \Sigma_{\mathrm{th}}\right), \\
\text { with } \Sigma_{\mathrm{th}, i j} & =\bar{c}^{2} y_{\mathrm{ref}, i} y_{\mathrm{ref}, j} \frac{Q_{i}^{k+1} Q_{j}^{k+1}}{1-Q^{2}} R_{i j} .
\end{aligned}
$$

Note that we use the same $Q_{i}=0.33$ for all $A=4,6$ observables.

\section{Full sampling of the PPD}

Results from sampling of the full PPD are shown in Fig. 8. In practice, we construct this full PPD with $\approx 2.5 \times 10^{6} \mathrm{sam}$ ples from the NCSM PPD with the addition of the random variables $\delta \boldsymbol{y}_{\mathrm{NCSM}}$ and $\boldsymbol{\delta} \boldsymbol{y}_{\mathrm{EFT}}$ sampled from the distributions (22) and (24), respectively. The sampling from the error distributions is simplified by the fact that the only LEC dependence sits in the mean value of the method error via the $\Delta E_{\infty}(\vec{a})$ term in Eq. (21). We are able to compute this extrapolation term for any set of $\vec{a}$ since we have created accurate emulators for $N_{\max } \in\{4,6,8,10\}$.

The separation energies, $S_{d}$ for ${ }^{6} \mathrm{Li}$ and $S_{2 n}$ for ${ }^{6} \mathrm{He}$, and the ${ }^{6} \mathrm{He}_{\mathrm{gs}} \rightarrow{ }^{6} \mathrm{Li}_{\mathrm{gs}}$ beta-decay $Q$ value are also included. Predictions for these relative observables will now make sense since we have incorporated both method errors and correlated model discrepancies. The former contains information on the convergence of the many-body solver, while the latter embodies the EFT truncation error and makes sure that we do not overestimate its effect when propagated to differences of correlated observables. The final predictions for $A=6$ total energies, shown in the second and third columns of Fig. 8,

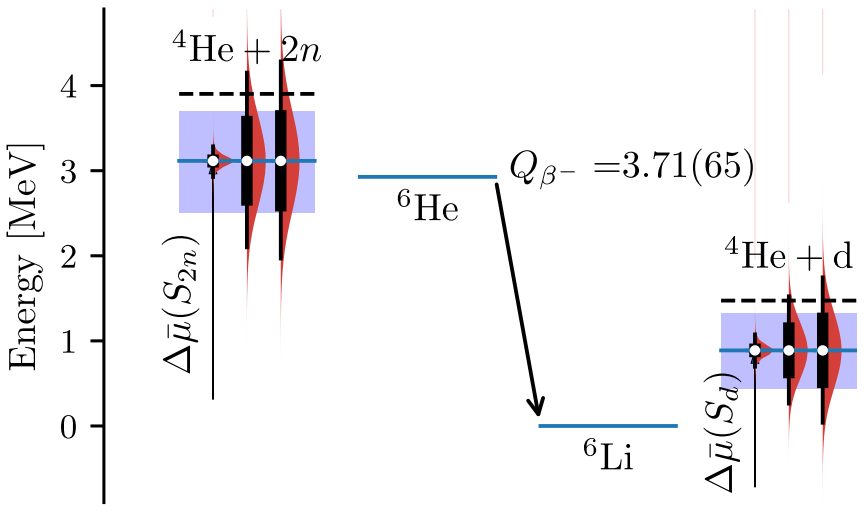

FIG. 9. $A=6$ level scheme. Dashed lines show experimental thresholds for ${ }^{4} \mathrm{He}+2 n\left({ }^{4} \mathrm{He}+d\right)$ relative ${ }^{6} \mathrm{He}\left({ }^{6} \mathrm{Li}\right)$ while the blue line and band show the median and $68 \%$ credible interval from the full PPD. The red distributions, from left to right, show the evolution of the PPD as we go from the NCSM prediction, $\mathrm{PPD}_{\mathrm{NCSM}}$, to the inclusion of method errors, and finally including the EFT truncation error-with thick (thin) vertical lines indicating the 68\% (95\%) credible interval. Note that the NCSM prediction for each threshold has been shifted by the mean values of the relevant method errors. The uncertainty in the $\beta^{-}$-decay $Q$ value is dominated by the method $\left(N_{\max }\right.$ extrapolation) uncertainty.

provide hints for systematic underbinding. We speculate that there is a possibility that nuclear interactions that are constructed using more relaxed low-energy constraints (causing the deuteron energy to be less accurately reproduced) can lead to systematic underbinding in larger systems. However, the statistical evidence for this proposition is not very significant.

We also employed a low-statistics set of samples with the resulting full PPD shown by the open, grey histograms in the diagonal panels of Fig. 8. Here we start from just 25 samples from $\mathrm{pr}_{\text {full }}\left(c_{D}, c_{E}\right)$ and the corresponding NCSM predictions. We then resample 25000 times from these 25 predictions and add samples from the method and model errors. The close resemblance of the resulting marginal PPD distributions with the high-statistics version shows that in this case it is indeed possible to propagate errors and extract relevant uncertainty quantification estimates also with a relatively small number of model predictions.

Finally, we show the $A=6$ level scheme in Fig. 9 with relevant uncertainties. This result demonstrates the precision that can be expected in our ab initio approach. The sequences of PPD distributions show that it is in fact the uncertainty in the many-body solver, and the process of extrapolating to infinite model space, that is responsible for the main fraction of the total error budget. This situation might be different for other many-body systems and using other many-body methods. We expect that already an extension to $N_{\max }=10,12$ for ${ }^{6} \mathrm{He}$ and $N_{\max }=12$ for ${ }^{6} \mathrm{Li}$ would significantly reduce these method errors.

\section{SUMMARY AND OUTLOOK}

The main findings and conclusions of this study are as follows: 
(i) This work introduces JUPITERNCSM-a new $M$ scheme NCSM code that uses on-the-fly Hamiltonian matrix construction in a SD basis with full inclusion of 3NFs. This code performs computationally efficient diagonalization using the Lanczos algorithm and can employ various convergence criteria. In particular, we perform single-node computations up to $N_{\max }=10$ for ${ }^{6} \mathrm{Li}$ focusing on obtaining well-converged eigenvectors.

(ii) EC emulators are constructed for $A=4,6$ systems using $M$-scheme NCSM eigenstates as training data. We construct emulators for ${ }^{4,6} \mathrm{He}$ and ${ }^{6} \mathrm{Li}$ in different model spaces from $N_{\max }=4$ up to $N_{\max }=10$. After the training phase, the emulators provide a computational speedup reaching seven orders of magnitude with very high output accuracy. In fact, we demonstrate that emulated binding energies are accurate to within $\lesssim 10^{-7}$ relative error in a large $c_{D}-c_{E}$ parameter domain with only 8-16 training points.

(iii) This work demonstrates a Bayesian approach for handling relevant sources of uncertainty in manybody nuclear structure calculations. We consider uncertainties in (i) the parametrization of the nuclear interaction, (ii) the model discrepancy arising from the truncation of the $\chi$ EFT expansion, and (iii) the solution of the many-body problem. This study is made possible by employing Bayesian methods and using EC emulator technology and the newly developed and efficient JUPITERNCSM many-body solver.

(iv) Realistic and statistically rigorous constraints from few-nucleon observables lead to quantifiable propagated uncertainties in $A=6$ systems. We employ Bayesian constraints on chiral three-nucleon forces from few-body observables as quantified by Wesolowski et al. [6]. The parameter posterior PDF from that study is characterized by a strong correlation and rather heavy tails as a result of a full treatment of EFT truncation uncertainties. These features are also reflected in the propagated parameter uncertainty for $A=6$ observables. Although our study of parameter uncertainty is limited to $c_{D}$ and $c_{E}$-parameters of the leading three-nucleon force-we have hints in ${ }^{4} \mathrm{He}$ that propagated uncertainties from $\pi \mathrm{N}$ and NN LECs are small in comparison.

(v) A possible separation of modes in the posterior predictive distribution for finite nuclei is observed when using an alternative force calibration. It is well known that correlated calibration observables lead to insufficient constraints on model parameters. Using, e.g., binding energies of ${ }^{3} \mathrm{H}$ and ${ }^{4} \mathrm{He}$ to calibrate the $c_{D}, c_{E}$ parameters of the $3 \mathrm{NF}$ gives an effective constraint in just a single direction. We find that binding energies of $A=6$ systems are also correlated-such that they would not offer a strong complimentary constraint - but there are indications for a separation into two modes implying that effects in heavier nuclei might be large. (vi) The inclusion of an EFT model discrepancy term is important for proper uncertainty quantification. Observed order-by-order predictions corroborate a view of converging nuclear structure observables and allows us to construct a statistical model for the discrepancy originating from the truncation of the EFT expansion. We find that the magnitudes of observable coefficients $\bar{c}$ are consistent with previous works. In the end we employ a simplified model with fixed hyperparameters and recommend further statistical analysis to learn about the EFT convergence pattern. Furthermore, the Bayesian approach advocated here allows us to also incorporate knowledge of, e.g., the residual cutoff dependence of the EFT error, as studied for example by Yang et al. [30].

(vii) The treatment of correlated errors is needed for proper estimates of uncertainties in separation energies. We find compelling evidence that order-byorder contributions to the binding energies in $A=$ 4, 6 nuclei are strongly correlated. Assuming a single correlation coefficient to describe the observed convergence pattern we infer the most likely value $\rho=0.9$ which is then used in the uncertainty analysis. As a result, the final uncertainty in separation energies is better estimated. The uncertainties of these observables would have been severely overestimated without accounting for correlated errors.

(viii) Method errors are the dominating source of uncertainty in this study. The uncertainty of the manybody solution could be reduced by extending the model space beyond $N_{\max }=10(8)$ for ${ }^{6} \mathrm{Li}\left({ }^{6} \mathrm{He}\right)$. We note that computations up to $N_{\max }=22$ have been performed for ${ }^{6} \mathrm{Li}$ resulting in energy convergence at the level of $10 \mathrm{keV}$ [20]. However, three-body force approximation schemes (with proper uncertainty quantification) will be needed to reach such large model spaces.

(ix) Our study provides hints for systematic underbinding in $A=6$ systems. The observed underbinding is just at the edge of the $68 \%$ credible regions for both ${ }^{6} \mathrm{Li}$ and ${ }^{6} \mathrm{He}$ implying very weak evidence for possible physics interpretations of this finding. Futhermore, the $N_{\max }$ extrapolations that are employed in this work systematically overestimate the energy convergence rate-an observation that we have tried to take into account in our statistical error model. Still, it might be relevant to use infrared extrapolation techniques [20,23-25] to achieve a more systematic treatment and better understanding of method uncertainties.

\section{ACKNOWLEDGMENTS}

We thank P. Navrátil for useful discussions and for making it possible to perform validation of results from JUPITERNCSM and NCSD. This work was supported by the Swedish Research Council, Grant No. 2017-04234 (T.D., C.F.), and the European Research Council (ERC) European Unions Horizon 2020 research and innovation programme, Grant Agreement 
No. 758027 (A.E.). Parts of the computations were enabled by resources provided by the Swedish National Infrastructure for Computing (SNIC) at Chalmers Centre for Computational
Science and Engineering (C3SE) and the National Supercomputer Centre (NSC) partially funded by the Swedish Research Council.
[1] M. R. Schindler and D. R. Phillips, Bayesian methods for parameter estimation in effective field theories, Ann. Phys. 324, 682 (2009).

[2] R. J. Furnstahl, N. Klco, D. R. Phillips, and S. Wesolowski, Quantifying truncation errors in effective field theory, Phys. Rev. C 92, 024005 (2015).

[3] S. Wesolowski, N. Klco, R. J. Furnstahl, D. R. Phillips, and A. Thapaliya, Bayesian parameter estimation for effective field theories, J. Phys. G 43, 074001 (2016).

[4] S. Wesolowski, R. J. Furnstahl, J. A. Melendez, and D. R. Phillips, Exploring Bayesian parameter estimation for chiral effective field theory using nucleon-nucleon phase shifts, J. Phys. G 46, 045102 (2019).

[5] J. A. Melendez, R. J. Furnstahl, D. R. Phillips, M. T. Pratola, and S. Wesolowski, Quantifying correlated truncation errors in effective field theory, Phys. Rev. C 100, 044001 (2019).

[6] S. Wesolowski, I. Svensson, A. Ekström, C. Forssén, R. J. Furnstahl, J. A. Melendez, and D. R. Phillips, Rigorous constraints on three-nucleon forces in chiral effective field theory from fast and accurate calculations of few-body observables, Phys. Rev. C 104, 064001 (2021).

[7] K. Kravvaris, K. R. Quinlan, S. Quaglioni, K. A. Wendt, and P. Navratil, Quantifying uncertainties in neutron- $\alpha$ scattering with chiral nucleon-nucleon and three-nucleon forces, Phys. Rev. C 102, 024616 (2020).

[8] P. Maris, E. Epelbaum, R. J. Furnstahl, J. Golak, K. Hebeler, T. Huther, H. Kamada, H. Krebs, Ulf-G. Meissner, J. A. Melendez, A. Nogga, P. Reinert, R. Roth, R. Skibinski, V. Soloviov, K. Topolnicki, J. P. Vary, Y. Volkotrub, H. Witala, and T. Wolfgruber, Light nuclei with semilocal momentum-space regularized chiral interactions up to third order, Phys. Rev. C 103, 054001 (2021).

[9] C. Drischler, R. J. Furnstahl, J. A. Melendez, and D. R. Phillips, How Well Do We Know the Neutron-Matter Equation of State at the Densities Inside Neutron Stars? A Bayesian Approach with Correlated Uncertainties, Phys. Rev. Lett. 125, 202702 (2020).

[10] C. Drischler, J. A. Melendez, R. J. Furnstahl, and D. R. Phillips, Quantifying uncertainties and correlations in the nuclear-matter equation of state, Phys. Rev. C 102, 054315 (2020).

[11] T. Djärv, C. Forssén, A. Ekström, and H. T. Johansson, JupiterNCSM, GPLv2 (2021), https://github.com/ thundermoose/JupiterNCSM.

[12] D. Frame, R. He, I. Ipsen, D. Lee, D. Lee, and E. Rrapaj, Eigenvector Continuation with Subspace Learning, Phys. Rev. Lett. 121, 032501 (2018).

[13] S. König, A. Ekström, K. Hebeler, D. Lee, and A. Schwenk, Eigenvector continuation as an efficient and accurate emulator for uncertainty quantification, Phys. Lett. B 810, 135814 (2020).

[14] J. A. Melendez, S. Wesolowski, and R. J. Furnstahl, Bayesian truncation errors in chiral effective field theory: nucleonnucleon observables, Phys. Rev. C 96, 024003 (2017).
[15] D. Siemens, J. Ruiz de Elvira, E. Epelbaum, M. Hoferichter, H. Krebs, B. Kubis, and U. G. Meißner, Reconciling threshold and subthreshold expansions for pion-nucleon scattering, Phys. Lett. B 770, 27 (2017).

[16] B. D. Carlsson, A. Ekström, C. Forssén, D. F. Strömberg, G. R. Jansen, O. Lilja, M. Lindby, B. A. Mattsson, and K. A. Wendt, Uncertainty Analysis and Order-by-Order Optimization of Chiral Nuclear Interactions, Phys. Rev. X 6, 011019 (2016).

[17] E. Caurier and F. Nowacki, Present Status of Shell Model Techniques, Acta Phys. Pol. B 30, 705 (1999).

[18] P. Navrátil and E. Caurier, Nuclear structure with accurate chiral perturbation theory nucleon-nucleon potential: Application to ${ }^{6} \mathrm{Li}$ and ${ }^{10} \mathrm{~B}$, Phys. Rev. C 69, 014311 (2004).

[19] A. Ekstrom, G. Baardsen, C. Forssen, G. Hagen, M. HjorthJensen, G. R. Jansen, R. Machleidt, W. Nazarewicz, T. Papenbrock, J. Sarich, and S. M. Wild, Optimized Chiral Nucleon-Nucleon Interaction at Next-to-Next-to-Leading Order, Phys. Rev. Lett. 110, 192502 (2013).

[20] C. Forssén, B. D. Carlsson, H. T. Johansson, D. Sääf, A. Bansal, G. Hagen, and T. Papenbrock, Large-scale exact diagonalizations reveal low-momentum scales of nuclei, Phys. Rev. C 97, 034328 (2018).

[21] A. Ekström, G. R. Jansen, K. A. Wendt, G. Hagen, T. Papenbrock, B. D. Carlsson, C. Forssén, M. Hjorth-Jensen, P. Navrátil, and W. Nazarewicz, Accurate nuclear radii and binding energies from a chiral interaction, Phys. Rev. C 91, 051301(R) (2015).

[22] P. Navrátil, No-Core Shell Model Slater Determinant code (NCSD) (unpublished).

[23] S. A. Coon, M. I. Avetian, M. K. G. Kruse, U. van Kolck, P. Maris, and J. P. Vary, Convergence properties of $a b$ initio calculations of light nuclei in a harmonic oscillator basis, Phys. Rev. C 86, 054002 (2012).

[24] R. J. Furnstahl, G. Hagen, and T. Papenbrock, Corrections to nuclear energies and radii in finite oscillator spaces, Phys. Rev. C 86, 031301(R) (2012).

[25] K. A. Wendt, C. Forssén, T. Papenbrock, and D. Sääf, Infrared length scale and extrapolations for the no-core shell model, Phys. Rev. C 91, 061301(R) (2015).

[26] D. R. Tilley, H. R. Weller, and G. M. Hale, Energy levels of light nuclei $A=4$, Nucl. Phys. A 541, 1 (1992).

[27] M. Wang, G. Audi, A. Wapstra, F. Kondev, M. MacCormick, $\mathrm{X}$. Xu, and B. Pfeiffer, The ame2012 atomic mass evaluation, Chin. Phys. C 36, 1603 (2012).

[28] D. K. Frame, Ab initio simulations of light nuclear systems using eigenvector continuation and auxiliary field Monte Carlo, Ph.D. thesis, Michigan State University, 2019.

[29] C. Forssén, J. P. Vary, E. Caurier, and P. Navrátil, Converging sequences in the ab initio no-core shell model, Phys. Rev. C 77, 024301 (2008).

[30] C. J. Yang, A. Ekström, C. Forssén, and G. Hagen, Power counting in chiral effective field theory and nuclear binding, Phys. Rev. C 103, 054304 (2021). 